\title{
Evaluation of the Expression of Genes Associated with Inflammation and Apoptosis in Androgenetic Alopecia by Targeted RNA-Seq
}

\author{
Lizeth Martinez-Jacobo a, b,f Claudia I. Ancer-Arellano ${ }^{c}$ Rocio Ortiz-Lopez ${ }^{\text {b, d }}$ \\ Mauricio Salinas-Santander ${ }^{\mathrm{e}}$ Cesar Daniel Villarreal-Villarreal ${ }^{c}$ Jesus Ancer-Rodriguez ${ }^{\mathrm{b}}$ \\ Bianka Camacho-Zamora ${ }^{a}$ b Viviana Zomosa-Signoret ${ }^{a}$ Carlos E. Medina-De la Garza ${ }^{b}$ \\ Jorge Ocampo-Candianic ${ }^{c}$ Augusto Rojas-Martinez ${ }^{b, d}$ \\ a Universidad Autonoma de Nuevo Leon, Departamento de Bioquímica y Medicina Molecular, Facultad de

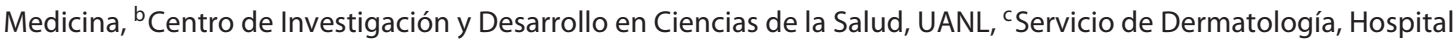 \\ Universitario, UANL, decnologico de Monterrey, Escuela de Medicina y Ciencias de la Salud, and e Universidad \\ Autonoma de Coahuila, Facultad de Medicina, Departamento de Investigacion, Saltillo, and ${ }^{\mathrm{f} U n i v e r s i d a d ~ d e}$ \\ Monterrey, Vicerrectoría de Ciencias de la Salud, Departamento de Ciencias Básicas, San Pedro Garza García, Mexico
}

\section{Keywords}

Androgenetic alopecia · Targeted RNA-Seq · Apoptosis · Inflammation

\begin{abstract}
Androgenetic alopecia (AGA) or male pattern baldness is the most common form of hair loss in humans. Despite being a very frequent dermatological entity, molecular pathophysiology remains unclear. Several authors relate the presentation of AGA with a premature apoptotic process during the anagen phase and with an inflammatory microenvironment in the hair follicle. We evaluated a panel of 30 genes associated with inflammation and apoptosis in 5 AGA patients by targeted RNA-Seq. WNT7A gene was highly expressed in patients in stages $3 \mathrm{~V}$ to 5 on the Hamilton-Norwood scale compared to patients with $5 \mathrm{~A}$ stage. CASP7 and TNF genes were overexpressed in stages $3 \mathrm{~V}$ and 4 compared to stages 5 and $5 A$. Overexpression of these genes detected only at early stages of AGA proves the role of WNT pathway, apoptosis, and inflammation in the development of this disorder.
\end{abstract}

(c) 2017 S. Karger AG, Basel

\section{KARGER}

(๑) 2017 S. Karger AG, Basel

E-Mail karger@karger.com

www.karger.com/sad

\section{Introduction}

Androgenetic alopecia (AGA), or male baldness pattern androgenetic alopecia, is the most common form of hair loss in humans and affects $80 \%$ of Caucasian men and $40-50 \%$ of Caucasian women [1]. In AGA, there is an alteration of the hair growth cycle [2]. AGA is characterized by miniaturization of the hair follicle, a phenomenon that occurs due to a rapid change from the anagen phase to the catagen/telogen phase, which probably reflects an ongoing follicle apoptotic process [3]. During the hair growth cycle, there is an important interaction between growth factors such as cytokines, hormones, and neurotransmitters and their receptors [4]. However, it is still not clear if AGA results from disruption of proliferation or an increase in apoptosis in the hair follicle [5]. Despite this, one of the main targets of topic and systemic antioxidant therapies for AGA is apoptosis inhibition [6]. In fact, studies of drugs such as finasteride have shown decreased levels of caspases after 6 months of treatment in patients with AGA compared with healthy subjects [7]. Most studies evaluating caspases in patients with AGA 
Table 1. Clinical data of the studied groups

\begin{tabular}{lll}
\hline Variables & Patients & Controls \\
\hline Age range, years & $22-52(33.68 \pm 10.6)$ & $21-22(21.5 \pm 0.7)$ \\
Males, $n(\%)$ & $10(100)$ & $2(100)$ \\
Severity of disease (Hamilton-Norwood scale) & \\
1 & - & - \\
2 & - & - \\
$3 \mathrm{~V}$ & 2 & - \\
4 & 3 & - \\
5 & 2 & - \\
$5 \mathrm{~A}$ & 3 & - \\
Range of duration of & & 0 \\
$\quad$ disease, months & $24-360(108 \pm 93.6)$ & 0 \\
\hline
\end{tabular}

have been performed with immune-based techniques, such as immunohistochemistry and ELISA, to analyze gene expression. The purpose of this article was to evaluate the expression of a panel of genes associated with inflammation and apoptosis in AGA using targeted RNASeq.

\section{Material and Methods}

\section{Tissue Samples and RNA Isolation}

Ten patients with AGA (3V to $5 \mathrm{~A}$ ) and 2 control subjects were included in the study (Table 1). Clinical and dermatological evaluation was performed to confirm the diagnosis of AGA. Patients with diagnosis of seborrheic dermatitis, telogen effluvium, and other types of alopecias were excluded from this study. The Ethics and Research Committees of the School of Medicine and University Hospital of Universidad Autonoma de Nuevo Leon at Monterrey, Mexico, approved and registered the study protocol with the code BI14-001 to be conducted from January 2014 to August 2016. Two scalp biopsies of $4 \mathrm{~mm}$ were collected from each subject. Patients with AGA consisted of males aged from 18 to 40 years old, who had not been treated with finasteride or minoxidil. Biopsies were obtained from the affected area and the occipital region in these patients. Biopsies from control subjects were collected from the vertex and occipital regions. RNAlater ${ }^{\circledR}$ solution (Thermo-Fisher Scientific, Waltham, MA, USA) for RNA stabilization and storage was used to preserve biopsies. RNA from scalp biopsies was isolated with the RNeasy Blood and Tissue kit ${ }^{\mathrm{TM}}$ (QIAGEN, Hilden, Germany) and was quantified with the QuantiT TM RiboGreen $^{\circledR}$ RNA Assay kit (Thermo-Fisher Scientific). Samples collected for this study were carefully preserved and stored at $-80^{\circ} \mathrm{C}$ until use to maintain RNA integrity before the molecular analyses were performed. The RQI (RNA quality indicator) number was evaluated using the Experion ${ }^{\mathrm{TM}}$ automated electrophoresis system (Bio-Rad, Hercules, CA, USA). The cutoff for the analyzed RNA samples had values above 7.8. Values from 7 to 10 are considered of good quality for microarray analysis or sequencing [8].

Targeted Expression Analysis for AGA
Next-Generation Sequencing

RNA (100 ng) from 5 patients (biopsies from affected and occipital areas) and 2 control subjects was used to obtain cDNA using the ProtoScript Reverse Transcriptase kit (New England Biolabs, Ipswich, MA, USA) and for the library preparation using TruSeq ${ }^{\circledR}$ Targeted RNA (Illumina, San Diego, CA, USA). Sequencing of 30 selected transcripts plus an endogenous GAPDH transcript control (Table 2) was carried out with MiSeq ${ }^{\circledR}$ Reagent Kit v3 (Illumina) (150 cycles) and Truseq ${ }^{\circledR}$ Target RNA Custom Panel Libraries (Illumina). Libraries were quantified using Quant-iT ${ }^{\mathrm{TM}}$ PicoGreen $^{\circledR}$ dsDNA Assay Kit (Thermo-Fisher Scientific). Targeted RNA-Seq data analysis was performed using TruSeq Targeted RNA v1.0 App in BaseSpace (Illumina). After demultiplexing and FASTQ file generation, readings of each sample were aligned against specified references in the manifest using a banded SmithWaterman alignment, which produces target hits files that contain raw aligned replicate counts for each transcript. Differential expression analysis was performed in which depth of sequencing normalization, variance estimation, and $p$ values were calculated. $p$ values reaching statistical significance for the differential expression were adjusted for false discovery rate (FDR) using the Benjamini-Hochberg method. A heat map was generated using Graph Pad Prism 7 (Graph Pad Software, Inc., La Jolla, CA, USA), considering the gene expression value as $\log _{10}$ of the number of readings for each gene per patient.

\section{Validation by Real-Time PCR}

Differential expression of CASP7, WNT7A, and TNF genes identified by targeted RNA-Seq was confirmed using real-time PCR analysis. The altered expression of WNT7A (ID: Hs01114990_ m1), CASP7 (ID: Hs00169152_m1), and TNF (ID: Hs00174128_ $\mathrm{m} 1$ ) genes in AGA were further confirmed in independent scalp biopsies, using AK3 (ID: Hs00750254_s1) as endogenous gene, based on the low variability observed in all samples tested by a previous microarray analysis. First strand $\mathrm{CDNA}$ was then synthesized from $2 \mu$ g total RNA using Superscript First Strand cDNA Synthesis Kit (Invitrogen, Carlsbad, CA, USA). Subsequently, real-time PCR reactions were performed using CDNA, TaqMan Universal PCR Master Mix, and Taqman Gene Expression Assays (ThermoFisher). The analysis was performed in a LightCycler 480 II RealTime PCR System (Roche Applied Science, Pleasanton, CA, USA), all according to the manufacturers' instructions.

\section{Statistical Analysis}

Comparison of numerical variables between the study groups was made using Student $t$ test for independent samples normally distributed. $p$ values $<0.05$ were considered significant. An unpaired Student $t$ test analysis was used to detect gene expression by real-time PCR analyses using GraphPad Prism 7 software.

\section{Results}

\section{Next-Generation Sequencing}

A panel of 30 genes associated with inflammation and apoptosis was performed for 5 patients with AGA and 2 controls by targeted RNA-Seq panel (Table 2). Higher expression of CASP3 $(p=0.0003), \operatorname{CASP7}(p=0.0001)$, 
Table 2. Genes included in the RNA-Seq panel

\begin{tabular}{|c|c|}
\hline Gene & Function \\
\hline TNF & $\begin{array}{l}\text { This cytokine is involved in the regulation of a wide spectrum of biological processes including } \\
\text { cell proliferation, differentiation, apoptosis, lipid metabolism, and coagulation }\end{array}$ \\
\hline CASP3 & $\begin{array}{l}\text { Is a member of the cysteine-aspartic acid protease (caspase) family; plays a central role in the } \\
\text { execution phase of cell apoptosis }\end{array}$ \\
\hline CASP7 & $\begin{array}{l}\text { Is a member of the cysteine-aspartic acid protease (caspase) family; plays a central role in the } \\
\text { execution phase of cell apoptosis/inflammation }\end{array}$ \\
\hline CASP8 & $\begin{array}{l}\text { Is a member of the cysteine-aspartic acid protease (caspase) family; plays a central role in the } \\
\text { execution phase of cell apoptosis }\end{array}$ \\
\hline CASP10 & $\begin{array}{l}\text { Is a member of the cysteine-aspartic acid protease (caspase) family; plays a central role in the } \\
\text { execution phase of cell apoptosis }\end{array}$ \\
\hline BAX & $\begin{array}{l}\text { BCL2-associated X protein, act as anti- or pro-apoptotic regulator that is involved in a wide } \\
\text { variety of cellular activities }\end{array}$ \\
\hline TNFRSF1A & $\begin{array}{l}\text { Is one of the major receptors for the tumor necrosis factor- } \alpha \text {; can activate NF- } \kappa \mathrm{B} \text {, mediate } \\
\text { apoptosis, and function as a regulator of inflammation }\end{array}$ \\
\hline TXN & Thioredoxin; inhibits oxidative stress and caspase 3 activity \\
\hline BCL2 & Encodes an integral outer mitochondrial membrane protein that blocks the apoptotic death \\
\hline POMC & $\begin{array}{l}\text { One peptide produced from the POMC protein is } \alpha-\mathrm{MSH} \text { involved in regulating the pigment- } \\
\text { producing cells of the skin and hair (melanocytes), where it binds to melanocortin } 1 \text { receptor } \\
(\mathrm{MC} 1 \mathrm{R}) / \text { inflammation }\end{array}$ \\
\hline $\mathrm{MC1R}$ & Controls melanogenesis/inflammation inhibitor \\
\hline MC4R & $\begin{array}{l}\text { Encoded protein interacts with adrenocorticotropic and MSH hormones and is mediated by G } \\
\text { proteins/inflammation inhibitor }\end{array}$ \\
\hline TPT1 & Microtubule stabilization/inflammation \\
\hline FASLG & Fas ligand (TNF superfamily, member 6); induction of apoptosis triggered by binding to FAS \\
\hline BCL3 & $\begin{array}{l}\text { Involved in regulation of cell proliferation and contributes to transcriptional regulation of } \\
\text { NFKB. Pro-survival and pro-inflammatory }\end{array}$ \\
\hline NFKB1 & $\begin{array}{l}\text { Inflammation/cell damage. Nuclear Factor Kappa B Subunit 1; is a transcription regulator } \\
\text { which stimulates the expression of genes involved in a wide variety of biological function }\end{array}$ \\
\hline TYRP1 & $\begin{array}{l}\text { Encodes a melanosomal enzyme that belongs to the tyrosinase family and plays an important } \\
\text { role in the melanin biosynthetic pathway. Inflammation }\end{array}$ \\
\hline DCT & Regulating eumelanin and phaeomelanin levels/inflammation \\
\hline MLANA & Involved in melanosome biogenesis/oxidative stress \\
\hline WNT7A & $\begin{array}{l}\text { Wnt Family Member 7A, implicated in oncogenesis and in several developmental processes. } \\
\text { Hair growth/inflammation inhibitor }\end{array}$ \\
\hline MITF & $\begin{array}{l}\text { Involved in melanocyte survival, controls the expression of genes related to melanin synthesis. } \\
\text { Cell differentiation, proliferation, and survival/inflammation }\end{array}$ \\
\hline MAPK1 & $\begin{array}{l}\text { Mitogen-activated protein kinase 1, involved in cell proliferation and differentiation/ } \\
\text { inflammation }\end{array}$ \\
\hline CAPN3 & Intracellular protease/activated in apoptotic cells \\
\hline CFLAR & Is a regulator of apoptosis and is structurally similar to caspase-8/apoptosis inhibitor \\
\hline CCBL2 & Cysteine conjugate-beta lyase 2 , involved in the regulation of oxidative stress/inflammation \\
\hline
\end{tabular}


Table 2 (continued)

\begin{tabular}{ll}
\hline Gene & Function \\
\hline CDC5L & Cell cycle control/inflammation \\
\hline CSNK1G3 & WNT signaling/inflammation \\
\hline PHACTR2 & Phosphatase and actin regulator 2/inflammatory cytokine in dermal cells \\
\hline GGT1 & $\begin{array}{l}\text { Involved in maintenance of intracellular GSH level. It is part of the cellular antioxidant defense } \\
\text { mechanism/inflammation }\end{array}$ \\
\hline GAPDH & Glyceraldehyde-3-phosphate dehydrogenase. Endogenous control/constitutive expression. \\
\hline GPD1 & Glycerol-3-phosphate dehydrogenase 1, involved in the redox metabolism \\
\hline GAPDH & Glyceraldehyde-3-phosphate dehydrogenase. Endogenous control/constitutive expression \\
\hline The function of the genes was extracted from: GeneCards Human Gene Database and UCSC Genome-Brows- \\
er.
\end{tabular}

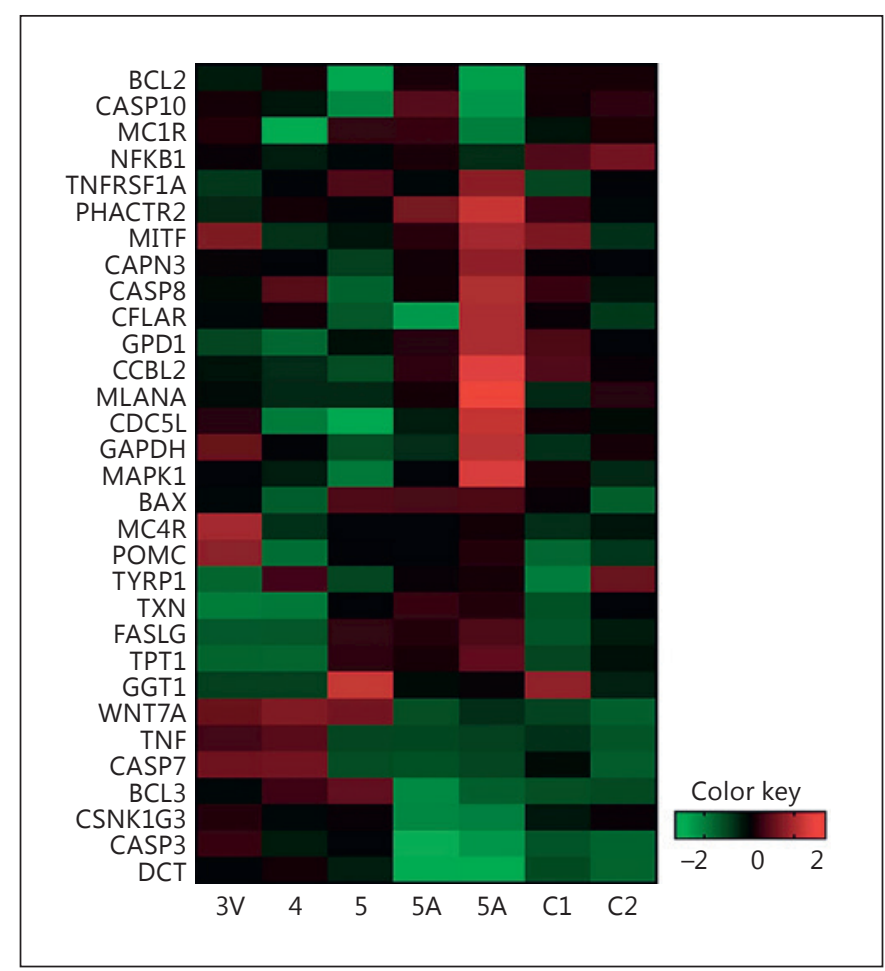

Fig. 1. Heat map of the profile of expression differences between the balding regions of patients with types $3 \mathrm{~V}$ to $5 \mathrm{~A}$ of androgenetic alopecia (Hamilton-Norwood scale). Genes with high expression are observed in red (upregulated), while those with low expression are represented in green (downregulated) $(p<0.05)$. Data was processed using the Graph Pad Prism 7 program. Each column represents a type of androgenetic alopecia according to the Hamilton-Norwood scale and each row represents a gene. The gene expression value corresponds to $\log _{10}$ of the number of readings for each gene per patient.

Targeted Expression Analysis for AGA
$B C L 3(p=0.0166), W N T 7 A(p=0.0005)$, and TNF $(p=$ 0.0004 ) was found in the affected area of patients with $3 \mathrm{~V}$, 4 , and 5; and underexpressed in 5A patients (Fig. 1).

\section{Validation by Real-Time PCR}

Targeted RNA-Seq analysis was confirmed using realtime PCR analyses for selected genes. Overexpression of CASP7 $(p=0.0001)$ and TNF $(p=0.0005)$ in patients with types $3 \mathrm{~V}$ and 4 was confirmed. Underexpression of these same genes was also confirmed in patients with types 5 and 5A. WNT7A ( $p=0.0001)$ was overexpressed in patients with types $3 \mathrm{~V}$ to 5 , whereas it was underexpressed in type $5 \mathrm{~A}$ patients. Differences were confirmed using unpaired Student $t$ test between two groups (3V-4 vs. 5-5A and $3 \mathrm{~V}-5$ vs. $5 \mathrm{~A}$ ) (Fig. 2). Expression analyses of CASP3 and $B C L 3$ were not evaluated due to limitations in sample availability.

\section{Discussion}

The role of the Wnt pathway in AGA is well established, as it is involved in the hair growth cycle and the morphogenesis of the hair follicle. Therefore, overexpression of WNT7A in moderate degrees (3V to 5) and its underexpression in the $5 \mathrm{~A}$ severe phenotype prove that in late stages of AGA, the hair growth cycle is suppressed, causing follicles to remain in the catagen/telogen phase. In this study, CASP7 and TNF showed overexpression in stages $3 \mathrm{~V}$ and 4 , but not in stages 5 and $5 \mathrm{~A}$ (Fig. 1). CASP7 belongs to the subgroup of executioner caspases, as does 


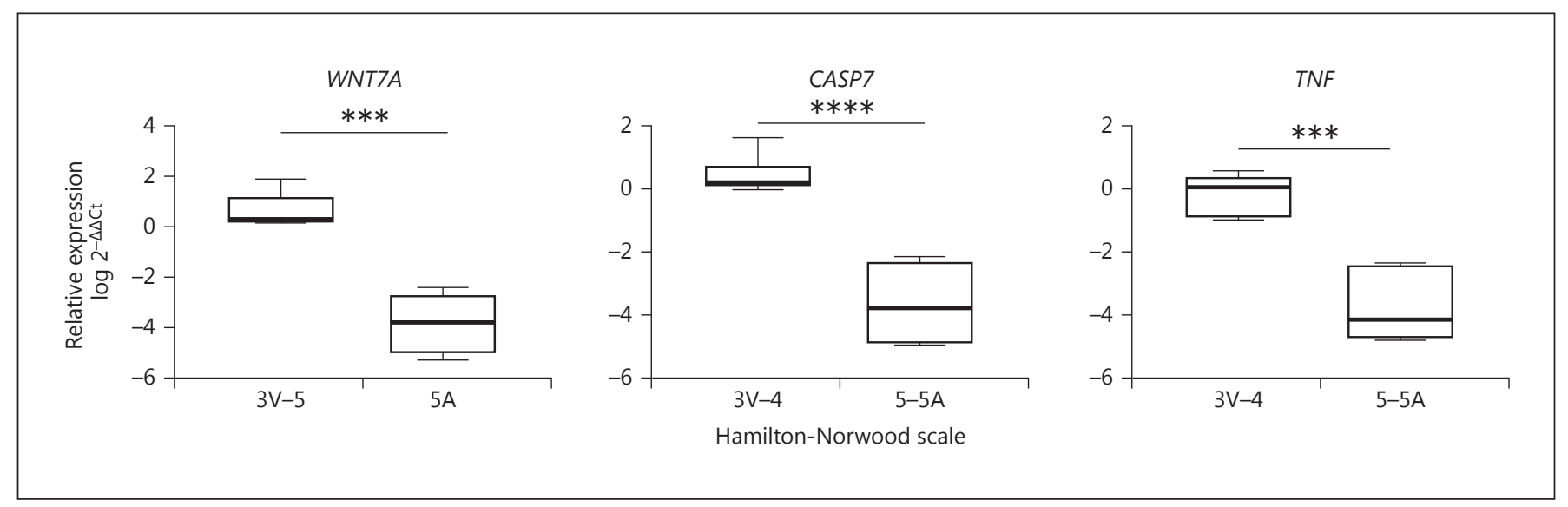

Fig. 2. Validation of the expression of WNT7, CASP7, and TNF genes by real-time PCR. Unpaired $t$ test was used to detect differences in gene expression between 2 groups (3V-4 and 5-5A types) using the Graph Pad Prism 7 software. Significance: ${ }^{* * *} p<0.001$ and ${ }^{* * * *} p<0.0001$.

CASP3 and CASP6. Interestingly, CASP7 proteolytic maturation is involved in apoptosis and inflammation, in contrast to $C A S P 3$, which participates in the apoptosis process only [9]. CASP7 expression is observed in cultured keratinocytes and in the anagen phase of the hair follicle in vivo $[10,11]$. Nevertheless, the role of CASP7 in the hair follicle is still controversial, since the gene remains active during the proliferation and differentiation processes in absence of evident apoptosis, suggesting additional roles for this enzyme [12].

Conversely, TNF is an inflammatory cytokine that plays a well-established role and acts on several different signaling pathways through two cell surface receptors, being probably the most potent inducer of apoptosis [13]. TNF has been recently added to the list of growth factors regulating hair follicle morphogenesis and has been traditionally associated with host defense, immunity, inflammation, and cancer [14]. TNF appears to protect against hair loss. The blockade of TNF by monoclonal antibodies resulted in alopecia areata in a patient treated with infliximab [15]. TNF overexpression in early phases of AGA and its downregulation in advanced stages of the disease suggest a protective effect for this protein.

Caspase- 3 plays an important role in apoptosis, as it is activated in the apoptotic cell by extrinsic (death ligand) and intrinsic (mitochondrial) pathways [16]. Overexpression of CASP 3 was only found in stages $3 \mathrm{~V}$ to 5 , indicating that this gene is involved in early stages of AGA. In contrast, CASP 3 is less expressed in advanced stages, probably reflecting the profuse destruction of hair follicles and the resulting perifollicular fibrosis. These results coincide with the report by Fawzi et al. [17], which stated that DKK1 levels, a WNT inhibitor, only influenced early stages of AGA. Overexpression of BCL3 in the clinical presentations $3 \mathrm{~V}$ to 5 of AGA may reflect a response of the affected hair follicles to CASP 3 expression, since elevated $B C L 3$ activity points towards increased cell proliferation and survival [18].

There are several limitations in our study. The sample size for each type of the Hamilton-Norwood scale needs to be increased to clarify CASP 3 and WNT7A overexpression at early stages, like in types $1-3$, where minimal bitemporal hair recession is evident. In addition, the number of transcripts analyzed in this study may result limited to study additional pathways involved in AGA progression. Due to the small number of samples, it was not possible to evaluate the expression of CASP 3 and BCL3 genes by real-time PCR, which were also overexpressed in types $3 \mathrm{~V}$ to 5 AGA patients. However, in this pilot study we proved the efficiency of molecular techniques as RNA-Seq and real-time PCR for the analysis of androgenetic alopecia, since to date, most studies evaluating caspases in patients with AGA have used immunohistochemistry and ELISA techniques to analyze the gene expression. It is also important to mention that the number of samples has been the limitation of other breakthrough studies in the AGA research field, since the diagnosis of this entity does not required a biopsy. Also, techniques such as microarray, sequencing, and real-time PCR generate a higher cost, compared with techniques of immunohistochemistry and ELISA. 
In conclusion, this study proves that $W N T 7 A, C A S P 7$, and $T N F$ are overexpressed in alopecia areas in patients with AGA at early stages of the disease. This result confirms the role of WNT pathway, apoptosis, and inflammation on early stages of this disorder. Targeted RNASeq is a useful technique for the evaluation of gene expression in AGA samples, as it only requires a small amount of input material for evaluating large numbers of genes.

\section{Acknowledgements}

We wish to thank all patients who generously participated in this study. This study was founded in part by the Centro de Investigación y Desarrollo en Ciencias de la Salud of the Universidad Autonoma de Nuevo Leon. Lizeth Martinez-Jacobo was supported by a CONACYT scholarship.

\section{Statement of Ethics}

The Ethics and Research Committees of the School of Medicine and University Hospital of Universidad Autonoma de Nuevo Leon at Monterrey, Mexico, approved and registered the study protocol with the code BI14-001 to be conducted from January 2014 to August 2016 .

\section{Disclosure Statement}

The authors declare that there is no conflict of interest that could be perceived as prejudicial to the impartiality of the reported research.

\section{References}

1 Heilmann S, Brockschmidt FF, Hillmer AM, Hanneken S, Eigelshoven S, Ludwig KU, Herold C, Mangold E, Becker T, Kruse R, Knapp M, Nothen MM: Evidence for a polygenic contribution to androgenetic alopecia. Br J Dermatol 2013;169:927-930.

2 Murphy GF: Mechanism of apoptotic regulation of follicular regression: toward understanding the molecular basis for tissue remodeling and regeneration. Am J Pathol 2001;158:1899-1901.

3 Hibino T, Nishiyama T: Role of TGF-beta2 in the human hair cycle. J Dermatol Sci 2004;35: 9-18.

4 Restrepo R: Anatomía microscópica del folículo piloso. Rev Asoc Colomb Dermatol 2010; 18:123-138.

5 Prieto VG, Sadick NS, Shea CR: Androgenetic alopecia: analysis of proliferation and apoptosis. Arch Dermatol 2002;138:1101-1102.

6 Guzmán-Sánchez DA: Alopecia Androgenética. Dermatol Rev Mex 2015;59:387-394.
7 Sawaya ME, Blume-Peytavi U, Mullins DL, Nusbaum BP, Whiting D, Nicholson DW, Lotocki G, Keane RW: Effects of finasteride on apoptosis and regulation of the human hair cycle. J Cutan Med Surg 2002;6:1-9.

8 Vladimir Denisov WS, Walder M, Gingrich J, Henri Wintz H: Development and Validation of RQI: An RNA Quality Indicator for the ExperionTM Automated Electrophoresis System. California, Bio-Rad Laboratories, 2008.

9 Lamkanfi M, Kanneganti TD: Caspase-7: a protease involved in apoptosis and inflammation. Int J Biochem Cell Biol 2010;42:21-24.

10 Takahashi T, Ogo M, Hibino T: Partial purification and characterization of two distinct types of caspases from human epidermis. J Invest Dermatol 1998;111:367-372.

11 Soma T, Ogo M, Suzuki J, Takahashi T, Hibino T: Analysis of apoptotic cell death in human hair follicles in vivo and in vitro. J Invest Dermatol 1998;111:948-954.

12 Vesela B, Svandova E, Vanden Berghe T, Tucker AS, Vandenabeele P, Matalova E: Non-apoptotic role for caspase-7 in hair follicles and the surrounding tissue. J Mol Histol 2015;46:443-455.
13 Zhang JM, An J: Cytokines, inflammation, and pain. Int Anesthesiol Clin 2007;45:27-37.

14 Gruss HJ, Dower SK: Tumor necrosis factor ligand superfamily: involvement in the pathology of malignant lymphomas. Blood 1995;85:3378-3404.

15 Fabre C, Dereure O: Worsening alopecia areata and de novo occurrence of multiple halo nevi in a patient receiving infliximab. Dermatology 2008;216:185-186.

16 Salvesen GS: Caspases: opening the boxes and interpreting the arrows. Cell Death Differ 2002;9:3-5

17 Fawzi MM, Mahmoud SB, Shaker OG, Saleh MA: Assessment of tissue levels of dickkopf-1 in androgenetic alopecia and alopecia areata. J Cosmet Dermatol 2016;15:10-15.

18 Chang TP, Vancurova I: Bcl3 regulates prosurvival and pro-inflammatory gene expression in cutaneous T-cell lymphoma. Biochim Biophys Acta 2014;1843:2620-2630. 